\title{
From cross to inter to trans - * cultural pragmatics on the move: The need for expanding methodologies in lingua franca research
}

\section{Introduction}

Pragmatics and intercultural communication are two interrelated areas that have been of crucial importance for ELF research, but are also entwined in multiple ways. Clearly, there is research on pragmatics that is not concerned with intercultural encounters, just like there is research on intercultural communication that is not centrally concerned with pragmatics. Nonetheless, pragmatics and intercultural communication show considerable overlap as research fields and have developed as closely connected areas over the past decades. Both of them have certainly shaped the way we investigate ELF communication.

This chapter reviews different perspectives on pragmatics - from cross- to interto transcultural - and explores implications for methodology that these different approaches to pragmatics have. ${ }^{1}$ It argues that the investigation of ELF interactions warrants a shift from inter- to transcultural pragmatics and that such a shift entails a change in methodologies used for analysing interactive data. Section 2 discusses pragmatics with a cross-cultural orientation and highlights methodological influences that this orientation tends to have (i.e. a focus on comparison). Section 3 looks at intercultural pragmatics and ELF communication and discusses its focus on analysing interaction with regard to three prevalent methodologies: interactional sociolinguistics, conversation analysis (CA) and corpus linguistics. Section 4 begins by arguing that the analysis of the pragmatics of (E)LF interactions, especially in transient contexts, prompts a conceptual shift from inter- to transcultural pragmatics. It argues that such a shift necessitates an expansion of methodologies in order to bring a potential transcultural pragmatic orientation to full fruition in descriptive research. It introduces such an expanded methodology by outlining principles of the micro-diachronic approach to spoken interaction. Section 5 summarises first findings on emergent multilingual practices gleaned through micro-diachronic analysis in three case studies in different ELF Transient International Groups.

1 The writing of this chapter was made possible as part of the Elise-Richter Grant 'English as a lingua franca in Transient International Groups' financed by the Austrian Science Fund (FWF): V747-G. 


\section{Pragmatics with a cross-cultural orientation: Data and methods for comparison}

Many influential theories and concepts in pragmatics, like Grice's (1989) cooperative principle, Austin and Searle's Speech Act theory (e.g. Austin 1975; Searle 1979) and Goffman's (1981) notion of face, started out with a first language (L1) ( English) focus. ${ }^{2}$ They were succeeded by an increased interest in cross-cultural comparisons across *languages in pragmatic research in the 1980s. Influential early examples include Blum-Kulka and Olshtain's (1984) Cross-Cultural Speech Act Realization Project (CCSARP) or Thomas's (1983) suggestions for cross-cultural pragmatic failure. These cross-cultural accounts of different L1 groups were in turn succeeded by a shift towards exploring pragmatics in contexts of intercultural exchanges in the 1990s and 2000s, as for example in Scollon and Scollon's (1995) research or Spencer-Oatey's (2002) work on rapport.

Although this shift from cross- to intercultural pragmatics, i.e. from the description of different L1 communities to the description of encounters among individuals with different L1 backgrounds, is noteworthy and influential for ELF research (see Section 3), the appearance of research on intercultural pragmatics has not led to a disappearance of the cross-cultural perspective. An example is the recently founded journal Contrastive Pragmatics (launched in 2020), which invites "contributions that compare the use of language forms, realisation of speech acts, forms of interactional behaviour, evaluative tendencies both across and historically within lingua-cultures [or] [. . .] pursue[. . .] interest in the contrastive study of patterns of translation and language teaching" (author instructions for Contrastive Pragmatics, emphasis mine). ${ }^{3}$ So although intercultural approaches to pragmatics may have historically appeared slightly after cross-cultural approaches and may have gained more traction in the past two decades (especially in relation to the study of ELF communication), this has not meant a complete abandonment of cross-cultural approaches.

Whether one adopts a cross- or intercultural approach - or maybe better: a cross- or intercultural orientation - to the study of pragmatics has direct implications for research methodology. It affects the data chosen for a study (see e.g. Zhu

\footnotetext{
2 The terms *language/s and *variety/ies and labels for individual *languages are written with an ${ }^{\star}$ symbol to convey a post-structuralist understanding of these terms that emphasise their non-boundedness and non-homogeneity.

3 The quoted passage is taken from a paragraph describing the scope of the journal in the online instructions for authors available at https://brill.com/fileasset/downloads_products/Author_Instructions/JOCP.pdf (accessed on 29 October 2021).
} 
2011: 4-8; Cutting 2015: 68-87) and is even more pertinent for the methods used to analyse pragmatic phenomena in data. While studies with a cross-cultural orientation tend to contrast data from different (often L1) speech communities, intercultural pragmatics and ELF research tend to examine interactions and encounters among multilingual speakers with different regio-cultural ${ }^{4}$ backgrounds (see Section 3 on intercultural pragmatics).

Cross-cultural pragmatic research prototypically involves comparing pragmatic conventions in two or more data sets taken from different *languages and/ or L1 speech communities (like in Blum-Kulka and Olshtain's 1984 influential CCSARP). In addition to describing pragmatic conventions in different *languages on the basis of L1 use, this can also involve comparing data from different groups of L2 (second language) users, learners or so-called non-native speakers. Notably, cross-cultural studies tend to collect L1, L2 or learner data from fairly homogenous samples (e.g. L1 users living in the same country/region or 'foreign language learners' with the same L1 background and/or the same age). The aim tends to be to compare these samples of pragmatic use with each other (e.g. L1 *English vs. L1 *Chinese vs. L1 ${ }^{\star}$ Swedish) or with L1 data of the same ${ }^{\star}$ language, i.e. comparing non-native/learner/L2 pragmatic conventions of a particular learner group with native/target language/L1 pragmatic conventions of the same *language. Pragmatic research situated within or closely related to SLA (Second Language Acquisition) often makes such comparisons between so-called native/ L1/target language behaviour and non-native/L2/learner pragmatic behaviour. In doing so, it adopts a perspective and terminology that is clearly quite different from research on ELF pragmatics. So although labels like L2 pragmatics, interlanguage pragmatics or target language pragmatics (see e.g. Cohen 2018) are not identical to cross-cultural pragmatics, they may share a (more or less pronounced and more or less explicit) cross-cultural (i.e. comparative and contrastive) orientation that is less commonly found in intercultural studies. ${ }^{5}$

4 Since the term culture can refer also to small cultures in the sense of Holliday (1999), i.e. including categories such as profession, institution, age and gender, I use the terms regio-culture/ regio-cultural when referring to large cultures in Holliday's (1999) sense.

5 Terminology and labels used to denote different stands of pragmatic research are quite messy, so there will always be exceptions to the general arguments made in this chapter. A general distinction between cross- and intercultural pragmatics along the lines outlined here seems warranted, but it might be better to conceive of this difference as a distinction between a cross- vs. an intercultural orientation in pragmatic research. Oftentimes, such a cross- vs. intercultural orientation will coincide with the terminology used by a scholar (i.e. cross-cultural / interlanguage / target language / L2 pragmatics vs. intercultural pragmatics). Nonetheless, the label(s) chosen by an individual scholar or in a particular publication may not match the general crossor intercultural orientation of the research. That is to say, although research may operate with 
In addition to choosing data that allow cross-cultural comparisons, pragmatic research with a cross-cultural orientation also relies on methods of analysis that enable such comparisons. Needless to say, the focus of analysis (i.e. what is actually compared) as well as the methods used for comparison can cover a wide spectrum. To use two recent examples (taken from the inaugural issue of Contrastive Pragmatics), pragmatic research with a cross-cultural orientation may draw on parallel corpora in order to contrast the pragmatic use of a word in different *languages (see Aijmer 2020 on *English absolutely vs. Swedish absolut) or employ discourse analysis to examine the perlocutionary effects of a particular public speech act in news media in different countries and different *languages (see Kramsch 2020).

The list of methods used for investigating cross-cultural pragmatic similarities and differences is clearly much longer. Importantly, it does not only involve the analysis of $3^{\text {rd }}$ person data in corpora (as in Aijmer 2020) or media texts (as in Kramsch 2020), but also includes elicitation techniques. A prime example is the well-established Discourse Completion Task (DCT) that already dates back to Blum-Kulka and Olshtain (1984). DCTs are not only employed for making cross-cultural pragmatic comparisons across L1s (for instance, concerning speech act realisations), but have also been particularly influential in interlanguage and L2 pragmatics.

Yet, as Golato's (2003) study of compliment responses demonstrates, DCT data and naturally-occurring interactive data actually yield considerably different results. Even if the stimuli (in Golato's study: compliments) used in a DCT are verbatim identical to naturally-occurring use and seek to re-create the naturally-occurring context, the compliment responses elicited in a DCT are considerably different from real-life responses (Golato 2003: 91). As an elicitation technique, DCTs are thus

in a crucial sense metapragmatic in that they explicitly require participants not to conversationally interact, but to articulate what they believe would be situationally appropriate responses within possible, yet imaginary, interactional settings. As such, responses within a DCT can be seen as indirectly revealing a participant's accumulated experience within a given setting, while bearing questionable resemblance to the data which actually shaped that experience.

(Golato 2003: 92, emphasis mine)

the label intercultural (as in intercultural communication or intercultural pragmatics), it may in fact exhibit a predominantly cross-cultural (i.e. comparative) orientation in research design. Conversely, research on L2 or interlanguage pragmatics may on occasion build upon or integrate a more intercultural (rather than cross-cultural) approach than one might expect on the basis of the terminology used. 
As criticised by the author, this difference between DCTs (and other elicitation techniques like role plays) and naturally-occurring interactions becomes particularly problematic if data from DCTs are used to make claims about "actual language use" (Golato 2003: 91). For most ELF researchers, who put a prime on naturally-occurring interactive data, this seems blatantly obvious. Clearly, elicited $2^{\text {nd }}$-person evidence cannot substitute $3^{\text {rd }}$-person naturally-occurring evidence and be used to make statements about it. Yet, claims about patterns of 'actual use' seem to be made quite regularly on the basis of elicited evidence in interlanguage pragmatics (see Golato 2003 for a more extensive critical discussion of this issue).

Similar points about methodology are also made by Bardovi-Harlig (2010), who speaks out in favour of analysing "samples of authentic and consequential language use [. . .] whenever possible” (Bardovi-Harlig 2010: 242). Her analysis of 152 publications in the field of interlanguage pragmatics concludes that only 41 (i.e. $27 \%$ ) of these actually "collected and analysed authentic language samples” (Bardovi-Harlig 2010: 241), although "[g]iven the focus of pragmatics research, this should be the default design for studies of production" (Bardovi-Harlig 2010: 242). Bardovi-Harlig's (2010) analysis shows that interaction and spoken language play a key role in interlanguage pragmatic research, but clearly demonstrates that the means (i.e. the data and methods) by which spoken interaction is examined in interlanguage pragmatics are sometimes highly questionable. For instance, 51 out of 57 studies that examine written data actually use these written data to make claims about spoken language (Bardovi-Harlig 2010: 237), which leads the author to demand, quite explicitly, that researchers "abandon [relying on] written production as a facsimile of oral production" (Bardovi-Harlig 2010: 242).

To summarise, the cross-cultural orientation of interlanguage, target language, L2 and cross-cultural pragmatics involves making comparisons on the basis of contrastive data (i.e. samples from different *languages and/or fairly homogenous samples of so-called non-native/L2 users or learners). It tends to employ methods of analysis that enable such comparisons. Yet, even within interlanguage pragmatics, researchers like Golato (2003) and Bardovi-Harlig (2010) urge caution concerning the claims made on the basis of elicited (contrastive) evidence. They emphasise the need for analysing naturally-occurring interactive data and point towards the use of conversation analytic methods in this respect (Golato 2003: 98-105; Bardovi-Harlig 2010: 241). Such naturally-occurring interactive data - and related methods of analysis - have, of course, shaped research on intercultural pragmatics and are prominent in the study of ELF pragmatics. 


\section{Intercultural pragmatics and ELF communication: Methods for analysing interaction}

In contrast to many studies with a cross-cultural orientation, intercultural pragmatics and research into the pragmatics of ELF communication exhibit a strong focus on interaction and thus naturally-occurring interactive spoken data. Both, intercultural and lingua franca research tend to examine encounters among multilingual speakers with different regio-cultural backgrounds. Building in particular on the work of Gumperz (1982), a prominent strand of intercultural communication research (and intercultural pragmatics) emerged as closely connected to interactional sociolinguistics in the 1980s and 1990s. Because of its inherent focus on interaction, methods of intercultural communication research and interactional sociolinguistics have had considerable influence on the investigation of ELF communication. ${ }^{6}$ Concepts like Sarangi's (1994) warning against an analytic stereotyping of intercultural communication, Holliday, Hyde and Kullman's (2004; see also Holliday 1999) non-essentialist view of culture and Zhu's (2014 and 2015) work on interculturality are, to my mind, fully compatible with a truly intercultural (as in, a non-contrastive) orientation. They have certainly been key influences in my own work on ELF pragmatics, especially on the nature of understanding, non-understanding and negotiation of meaning in ELF interactions (see e.g. Cogo and Pitzl 2016; Pitzl 2005, 2010, 2015, and 2017).

A second prominent methodological influence for the study of intercultural and ELF pragmatics is conversation analysis (CA). Numerous descriptive ELF studies rely on CA methodology to some extent. Notably, ELF researchers tend to differ with regard to how closely they align themselves and their research with CA. There is descriptive work on ELF interactions that operates more 'traditionally' with CA methods, such as work by Kaur (e.g. 2011, 2012, and 2021), Jenks (e.g. 2013), Pietikäinen (e.g. 2014, 2018, and 2021), Santner-Wolfartsberger (e.g. 2015), Konakahara (e.g. 2015), and some publications by Björkman (e.g. 2017), Mortensen and Hazel (e.g. 2017) and Matsumoto (e.g. 2018). Yet, some ELF scholars (including myself) also draw more loosely on CA methods and "apply selected

\footnotetext{
6 Notably, the study of intercultural encounters can sometimes nonetheless be informed by a native/non-native or L1/L2 (and hence cross-cultural) orientation, namely if interactive data are studied primarily through the lens of being native/non-native interactions which are used to identify patterns or problems of L1/L2 pragmatic behaviour. Such a native/non-native orientation is largely absent from research on ELF interactions and thus not characteristic of the description of ELF pragmatics.
} 
elements of CA in their analysis of ELF data”, as Kaur (2016: 164) remarks. One representative is Cogo (e.g. 2009, 2012, 2016a, and 2016b), who states to have used conversation analysis conjointly with "an ethnographic perspective" (Cogo 2009: 258), which she explicitly relates to Gumperz's (1999) work on interactional sociolinguistics. Cogo draws on linguistic ethnography more extensively (sometimes in connection with CA) also with regard to data collection, making use of elicited interviews and focus group data (i.e. $2^{\text {nd }}$-person data) in addition to naturally-occurring conversational ELF data (see e.g. Cogo 2012). In addition, some of her work examines topics like attitudes and ideologies (e.g. Cogo 2016a and Cogo 2016b), which are not typical concerns for work in CA, but more closely linked to areas like sociolinguistics, applied linguistics and discourse analysis - and, of course, intercultural communication research.

As illustrated by Cogo's research, the nature and role of elicited data for the study of ELF pragmatics is thus different from the role of elicited DCT data in interlanguage pragmatics outlined in Section 2. If elicited $2^{\text {nd }}$-person evidence is used to supplement the analysis of third-person ELF interactions, this does not usually entail a comparative or contrastive orientation in ELF research. That is to say, research on ELF pragmatics - and possibly on the pragmatics of lingua franca (LF) interactions more generally - is concerned with uncovering processes and patterns of pragmatic meaning-making in communication in real-life multilingual contexts (see e.g. Seidlhofer 2011; Widdowson 2015). Adopting a truly intercultural (as opposed to a cross-cultural) perspective on lingua franca use thus requires us to approach and analyse intercultural interactions without pre-imposing L1/native standards and without focusing on assumed or expected L2/non-native pragmatic, linguistic or *cultural differences or deficits.

A third methodological strand that has certainly influenced the study of intercultural ELF pragmatics is corpus linguistics. Both corpus linguistics as well as CA are methodologies with strong, but very distinct research communities that hardly overlap outside ELF research. Interestingly, the two methodologies have considerable intersections in the study of ELF communication, however. Both corpus linguistics and CA are well represented in publications by ELF scholars (although less often combined in the same publication). These intersections of CA and corpus linguistics in the study of ELF communication are, I would argue, a consequence of the nature of the data examined in ELF research and of the methods used to describe and analyse them.

Concerning the nature of the data, a substantial amount of ELF data has been made publicly available through the existence of ELF corpora like VOICE (Vienna-Oxford International Corpus of English, see e.g. Seidlhofer 2001, 2010, 2012, and 2013; Breiteneder, Klimpfinger, Majewski and Pitzl 2009), ELFA (Corpus of English as a lingua franca in academic settings, see e.g. Mauranen 2003, 2006, 
and 2016) and ACE (Asian Corpus of English, see e.g. Kirkpatrick 2016; Wang 2021). What is crucial in this respect is the fact that the prototypical data used to build ELF corpora are audio-recordings and detailed transcripts of spoken interactions. Because of their labour- and cost-intensive nature, these kinds of data are, however, notoriously underrepresented in most other *English language (i.e. non-ELF) corpora, where corpus size and quick availability of data oftentimes take precedence over the time and resources needed to carry out qualitative data collection, field recordings and detailed transcription. ${ }^{7}$ In consequence, present-day corpus linguistics is primarily driven by quantitative concerns and most corpus linguists who work with non-ELF corpora have little or no need to engage with the principles of spoken interaction. Their work oftentimes has no intersections with CA or interactional sociolinguistics, simply because the corpora they analyse hardly confront them with spoken and interactive data.

The second aspect has to do with the methods used for analysing (interactive ELF) data. Descriptive studies based on ELF corpora (and sometimes on individually collected ELF data sets) have drawn on methods of corpus analysis to study and describe ELF use. A more overt orientation towards corpus linguistics can be seen in descriptions of lexico-grammatical characteristics, for example, by Mauranen (2006 and 2012), Metsä-Ketelä (2006 and 2016), Ranta (2006 and 2018), Carey (2013) and Osimk-Teasdale (2014). Such descriptions, although largely based on interactive ELF data and certainly useful for evolving our empirical understanding of ELF communication, tend to focus less on the interactive nature of intercultural ELF encounters. This does not mean that these corpus-oriented ELF studies entirely disregard the interactive dimension of their data, but simply that interaction is usually not their main focus. Seeing that traditional corpus methods like keyword-in-context (kwic) concordances or wordlists are not targeted towards the study of interaction, this is not particularly surprising.

Of course, there are examples of descriptive ELF studies that combine conversation analysis, corpus methods and a pragmatic research focus in various ways. For instance, Bjørge (2010) engages with the typical CA topic of backchanneling by making use of corpus-based methods in a self-collected set of naturally-occurring simulated BELF student negotiations. Other examples are Cogo and Dewey (2012) and Pitzl (2018a). Both books are primarily concerned with qualitative analyses of ELF interactions and extensively rely on and discuss extracts of conversational transcripts. Yet, both studies also selectively use concordance output

7 But see e.g. Love, Dembry, Hardie, Brezina, and McEnery (2017) on the spoken component of the new BNC (British National Corpus) 2014. 
to support their arguments. The list of studies that combine corpus linguistics, CA and a focus on ELF pragmatics in some way is certainly longer than the few examples mentioned here. The main point I wish to make, however, is that although initial intersections of these distinct methodologies (i.e. CA and corpus linguistics) exist in ELF research, a fuller integration of CA, corpus methods and interactional sociolinguistics is desirable for future research.

This need becomes even more pressing if we take into account that lingua franca interactions may, in fact, not only be conceived as instances of intercultural but transcultural communication. Taking this distinction of inter- vs. transcultural communication seriously, especially also for pragmatic research, means that we need to expand the methodologies we use for the description of lingua franca encounters. As I shall argue and illustrate below, such an expansion of methodologies does not only require a more principled integration of different methodological research traditions, like interactional sociolinguistics, CA and corpus linguistics. It should also come with a shift in perspective that abandons the assumption that synchronic (interactive) data and individual speaker output are actually stable.

\section{Investigating transcultural pragmatics}

\subsection{Multilingualism and post-structuralism in applied linguistics and ELF research: From inter- to transcultural}

Current discourses and research on multilingualism in applied linguistics have obvious relevance and intersections with research on ELF communication (see e.g. Hülmbauer and Seidlhofer 2013; Jenkins 2015). In the past decade or so, there has been a multiplicity of trans-, multi-, poly-, super- and post-discourses on topics like transcultural flows (Pennycook 2007), translingual practice (Canagarajah 2013), translanguaging (e.g. Creese and Blackledge 2010; García and Li 2014; Li 2018), superdiversity (Blommaert 2010; cf. Vertovec 2007), heteroglossia (e.g. Blackledge and Creese 2020) and linguistic repertoires in a framework of poststructuralism (e.g. Busch 2012 and 2017; cf. also Blommaert and Backus 2011). These discourses have not only affected current strands of applied linguistics and discourse analysis, but should also find increasing reflections in pragmatic research.

Publications of some ELF scholars (e.g. Cogo 2012 and 2018; Baker 2015; Jenkins 2015; Baker and Sangiamchit 2019) align quite closely with these post-structuralist discourses. Although this may not always be explicitly addressed, I would argue 
that many ELF scholars (though admittedly not all) at least implicitly subscribe to a post-modernist, post-structuralist view of language, communication and culture a view that sees *languages and *cultures as non-finite, non-bounded, inherently dynamic, emergent, unstable and heterogeneous. If we adopt such a post-structuralist approach to language and communication, many ELF interactions will not be intercultural, in the sense of happening between *cultures, because "it may not always be clear what cultures participants are in-between or 'inter' in intercultural communication” (Baker 2018: 26). As Baker (2018: 26) continues, “'trans', as in 'transcultural communication', provides a better metaphor with its association of across and through rather than between and the suggestion of transgressing borders".

What I am proposing in this chapter is that such a shift from an inter- to a transcultural view on ELF communication is highly desirable for our work on ELF communication in general, but especially for our understanding and empirical work on pragmatics in heterogeneous multilingual settings. Crucially, this shift towards transcultural pragmatics should not only be terminological and conceptual, but needs to have direct implications for the methodologies we use to describe communication. Just like the shift from cross-cultural to intercultural pragmatics entailed a shift from methods of comparison (Section 2) to methods for analysing interaction (Section 3), the suggested move towards transcultural pragmatics entails a further shift in methodology. We need to systematically expand our analyses of relatively short stretches or extracts of transcribed (ELF) interactions in order to describe the full micro-diachronic development of communication in multilingual groups across entire speech events. For this purpose, we need to devise methodologies that allow us to uncover and describe in empirical detail the situational and group-specific emergence of transcultural norms and pragmatic conventions in real-time interaction.

\subsection{Transient International Groups and the need for a micro-diachronic approach}

Micro-diachronic analysis is a methodological approach that is currently being developed to enhance the empirical description of communication in transient multilingual contexts. It is closely connected to ongoing work on Transient International Groups (TIGs) (Pitzl 2018b, 2019, and 2021; cf. also Pitzl 2016a and Pitzl 2018a) and also Transient Multilingual Communities (TMCs) (e.g. Mortensen 2017). Research on TIGs and TMCs shares a common interest in the study of multilingual lingua franca contexts in which participants are (fairly) newly acquainted and potentially only interact for a relatively short amount of time. This general 
aim is highly compatible with current trans-, multi-, poly-, super- and post-discourses in applied linguistics (see above). One of the aspects that make transient contexts extremely interesting is that they tend to be low on "a scale of semiotic sedimentation" (Mortensen 2017: 275) with regard to linguistic and social norms. The same would, to my mind, also apply to pragmatic conventions. Since TIGs and TMCs are best regarded as trans- rather than intercultural, pragmatic conventions are likely to be in-flux and potentially co-developed throughout and by means of interaction rather than simply mapped onto conversations as pre-determined fixed L1 or L2 constructs or mind-sets.

A core assumption is that participants in lingua franca (and other multilingual) encounters are going to interact on the basis of their entire individual multilingual repertoires (IMRs) (Pitzl 2016a; see also e.g. Blommaert and Backus 2011; Seidlhofer 2011; Busch 2012 and 2017; Jenkins 2015; Hülmbauer 2016 on repertoires), not just their 'L2'/'foreign-language' *English skills. This will be the case even in contexts where overt forms and linguistic behaviour may appear to be just ${ }^{\star}$ English on the surface (see Cogo 2021 on overt vs. covert resources). In drawing on their IMRs in interaction, groups of multilingual speakers are going to engage continuously in moments of conscious, but more often involuntary and unconscious, sharing (see Pitzl 2018b) - a sharing of linguistic, multilingual and all other semiotic resources. Crucially, this includes any pragmatic behaviour and pragmatic language use. This continuing process of interaction and sharing will lead to the gradual expansion of a TIG's shared situational Multilingual Resource Pool (MRP) as well as to the expansion of speakers' IMRs (cf. Pitzl 2018b). What makes the study of (transient) lingua franca contexts so interesting is that not only norms themselves but also "the norm centre will not be given; it will be a matter to be explored" (Mortensen 2017: 274) in the process of interaction.

If we take a transcultural and translingual view on communication (as suggested in Section 4.1), not only linguistic, social and pragmatic norms but also norm centres will thus, at least to some extent, be jointly developed and negotiated throughout interactions. Clearly, the prominence vs. relative absence of contextual factors such as power relations and external hierarchies are likely to influence which norms - or whose norms - might be privileged as being adopted (or enforced) as norm centres in a particular situation. This holds true especially for what are often referred to as high-stakes encounters in which power relations may be ostensibly unequal (see e.g. Guido 2012 and Guido 2016; Dorn, Rienzner, Busch, and Santner-Wolfartsberger 2014; Radinger 2018; Kappa 2019; Seidlhofer 2021). Crucially, how unequal power relations are enacted by interlocutors and which norms might end up being privileged (but also resisted) in what way and to what extent by individual interlocutors in a given situation are matters for empirical investigation. If we want to trace these developments in detail, we 
need methodological tools that allow us to do so. The challenge of studying (E)LF encounters as truly transcultural is, I would argue, therefore not only conceptual, but in fact also deeply methodological in nature. If we are willing to explore the joint real-time creation of translingual, translanguaging and transcultural spaces in both high-stakes as well as more symmetrical and/or casual lingua franca encounters among multilinguals, we need to expand our methodologies in ways that allow us to unearth and visualise these processes along entire conversations and in sequences of speech events. The micro-diachronic approach to analysing interaction seeks to do just that.

\subsection{Principles of micro-diachronic analysis}

Micro-diachronic analysis as proposed as part of the TIGs framework (Pitzl 2018b, 2019, and 2021) combines interactional sociolinguistics, discourse and conversation analysis with pragmatics, but also integrates techniques from corpus linguistics. Although it relies on methodological tools developed as part of ELF corpus building (such as the mark-up used for transcription in VOICE), micro-diachronic analysis is not a corpus approach in a narrow sense. It constitutes an attempt to provide better research tools that allow us to highlight and portray the group and development dimension of interactions. Although this can involve the analysis of data extracted from corpora (as in the three studies on emergent multilingual practices summarised below), the micro-diachronic approach is not geared towards the analysis of entire corpora and it is not intended for making comparisons between ELF and L1 corpora. Instead, it is designed to be applicable to the analysis of individual speech events and clusters or sequences of speech events that involve the same interactants. Such speech events can be accessible as part of existing corpora (like VOICE, ACE or ELFA) or can be collected by individual scholars. Combining different methodologies (especially those outlined in Section 3), micro-diachronic analysis seeks to bridge the distinction between the description of usage vs. the description of use (see Widdowson 2021). It seeks to develop methodological tools that have the potential to integrate usage-based considerations, such as the role of frequency vs. salience (cf. e.g. Blommaert and Backus 2011; Schmid 2020), into detailed qualitative discourse analytic descriptions of pragmatic language use in conversational interactions.

In terms of data, the focus for the study of TIGs are naturally-occurring $3^{\text {rd }}$-person attested data, i.e. primarily speech events that have been audio- or video-recorded and subsequently transcribed in detail in their entirety (i.e. not just in selective portions). This emphasis on recorded $3^{\text {rd }}$-person data does not preclude multi-method approaches that might incorporate elicited $2^{\text {nd }}$-person 
evidence from interviews, focus groups or questionnaires (cf. e.g. Cogo's research summarised in Section 3). Yet, in contrast to most studies relying on a community of practice approach, the initial nexus of micro-diachronic analysis and the TIGs framework are naturally-occurring $3^{\text {rd }}$-person interactive data, not $2^{\text {nd }}$-person elicited evidence. The interactions currently used for developing tools for micro-diachronic analysis are transcribed spoken face-to-face encounters (see the case studies below), but the methodology can also be applied to the analysis of computer-mediated interactions (see Mujagic 2021).

What makes the suggested approach micro-diachronic is that detailed qualitative data analysis, manual and (semi-)automatic annotation of a linguistic phenomenon in interaction are combined with the close-meshed structural annotation of time segments and/or utterance sequences in interactive data. The combination of content and structural annotation and the use of QDA (qualitative data analysis) software make it possible to supplement traditional methods of data presentation in CA, interactional sociolinguistics and ELF research (such as the discussion of data extracts from transcripts) with novel tools and visualisations.

Such novel tools contextualise the examined phenomenon within the interaction as a whole (e.g. through overviews in tables or pie charts) and visualize chronological sequence by using micro-diachronic bar charts that represent entire interactions (see Pitzl 2021 and Pitzl in press). Both kinds of analytic tools can be used to investigate a particular linguistic phenomenon, such as the use of non-`English elements (see below). They can, however, also be employed to provide an "interaction and participation profile" (Pitzl 2021: 108) of interactive data. Pitzl (2021) and Pitzl (in press) provide such participation profiles, which offer numerical and visual information on the degree of active verbal participation by individual interlocutors. These interaction and participation profiles display the number of utterances of individual speakers holistically as well as micro-diachronically across the entire interaction (see Pitzl 2021: 106-108). Such tools offer information on the degree of interactivity of a speech event and provide details on the extent with which individual speakers do vs. do not actively verbally participate throughout an interaction or in different phases of a conversation or meeting.

This kind of information is extremely useful for the analysis of multi-party conversations (i.e. the prime data used in many ELF publications and ELF corpora), since it can provide concrete evidence that can be used to complement qualitative narrative accounts of how a speech event unfolds. Such ethnographic accounts are commonly found in ELF research and they remain valuable and necessary also within the suggested micro-diachronic approach. Thus, the proposed holistic and micro-diachronic visual tools should by no means simply replace 
detailed accounts of the data. Their purpose is to substantiate general observations and qualitative discussion of the data with concrete empirical evidence. Eventually, they have the potential to incorporate also aspects of group dynamics as a component of transcultural pragmatic analysis. If used more widely, interaction and participation profiles would make the qualitative findings gleaned on different interactive data more comparable across contexts and would provide a useful backdrop against with the prominence or salience (or relative frequency) of an investigated phenomenon can be discussed.

In addition, holistic and micro-diachronic visualizations can be used - in combination with the well-established practice of discussing data extracts - to investigate a particular phenomenon. The methodological tools of micro-diachronic analysis could, in principle, be used to describe emergent practices with regard to a wide range of linguistic, pragmatic, communicative and social phenomena. As summarised in the next section, micro-diachronic analysis has been used to describe emergent overt multilingual practices in an exemplary fashion in three different ELF-TIGs. This initial descriptive focus relates most immediately to the multilingual nature of ELF interactions as situations of transient language contact. The findings of these three case studies illustrate how we may be able to trace the potential evolution of situational norms in different ELF contexts.

\section{The micro-diachronic development of group- specific multilingual practices: Three case studies in ELF-TIGs}

This section summarises the use of non-^English elements in three ELF-TIGs as investigated in Pitzl (2018b, 2021, and in press). The three case studies demonstrate how different types of multilingual practices are developed by means of interaction in different TIGs over time. With regard to methodology, the discussion exemplifies the explanatory potential that micro-diachronic analysis can have in this respect.

The case studies examine emergent multilingual practices in three different ELF-TIGs recorded in different speech events in VOICE. Two of these groups take place in leisure contexts (Pitzl 2018b and in press), while a third TIG forms in a professional business context (Pitzl 2021). The interactions in all three TIGs have in common that the speakers use a fairly high number of non- ${ }^{\star}$ English elements as overt multilingual resources (to use Cogo's 2021 term) in comparison to other speech events in VOICE (see Pitzl 2021), but also in comparison to another 
ELF corpus like ELFA (cf. Hynninen, Pietikäinen, and Vetchinnikova 2017). The detailed micro-diachronic empirical descriptions of these TIGs demonstrate that non-`English elements function quite differently in the three groups. While the asymmetric-bilateral TIG in Malta investigated in Pitzl (2018b) and the diverse TIG of exchange students studied in Pitzl (in press) primarily develop different types of inclusive and shared overt multilingual practices in their casual conversations, the symmetric-bilateral business TIG in Pitzl (2021) gradually establishes tacit agreement concerning the use of 'exclusive' multilingual practices (in particular the use of unintelligible L1 side sequences).

\subsection{Case study 1: Establishing translingual and transcultural territory in an asymmetric-bilateral TIG}

To start with the more inclusive use of non-`English elements, Pitzl (2018b) shows how four multilingual speakers (three hosts and one visitor) establish translingual and transcultural territory. The micro-diachronic analysis traces how an initial discussion of local place and family names moves on to episodes of explicit language learning in which the visitor seeks to be taught words and phrases in the local *language (i.e. *Maltese, the hosts’ L1). In the course of three consecutive short speech events, the speakers establish increasing parallels between *Maltese and *Italian and, to a much lesser extent, also talk about ${ }^{\star}$ Serbian (i.e. the visitor's L1). The progression of these metalinguistic exchanges and episodes of language learning $\left(\mathrm{T}_{1} \rightarrow \mathrm{T}_{2}, \mathrm{~T}_{1} \rightarrow \mathrm{T}_{2}, \ldots\right)$ and the accompanying use of non ${ }^{\star}{ }^{*}$ English elements gradually creates an awareness in this ELF-TIG that (some knowledge of) ${ }^{\star}$ Italian is part of their shared Multilingual Resource Pool (MRP, cf. Pitzl 2016a).

This eventually prompts the visitor to use an ${ }^{\star}$ Italian idiom to express (a somewhat stereotypical) perceived similarity between people from the Mediterranean, loosely relating characteristics of people from Malta, Serbia, Italy and Turkey. Importantly, this is done by uttering the saying fuma come un turco as an overt code-switch in the original *language (i.e. *Italian), although this is neither participant's L1. *Italian can be said to function as a second, temporary lingua franca in this TIG, at least for the use of this idiom. Micro-diachronic analysis shows how the possibility of this particular multilingual practice is only gradually opened up by means of the preceding interaction in the group.

With the notable exception of the salient *Italian idiom, the asymmetric-bilateral TIG in Pitzl (2018b) almost exclusively contains non-^English elements from the local language (i.e. *Maltese), which are used by the hosts but also the visitor. The strong focus on just one other ${ }^{\star}$ language (i.e. ${ }^{\star}$ Maltese) in this 
bilateral TIG stands in contrast to the use of non- ${ }^{\star}$ English elements in the diverse TIG examined in Case study 2.

\subsection{Case study 2: Sharing multilingual resources and potential norm development in a diverse student TIG}

Although the TIG investigated in Pitzl (2018b) as well as the TIG interaction analysed in Case study 2 (Pitzl in press) take place in leisure contexts, the two groups are different in terms of group constellation. While the four speakers in Case study 1 constitute an asymmetric-bilateral TIG, the six students whose ELF interaction is analysed in Pitzl (in press) can be characterised as a linguistically diverse TIG. The participants in both leisure contexts exhibit a similar general interest in language learning, which is evidenced by episodes of explicit metalinguistic comments and explicit language teaching/learning in both case studies (see also Vettorel 2019). Yet, the different constellations of MRP/IMRs in the two groups (i.e. bilateral vs. diverse TIG) also prompt differences in their use of non- ${ }^{\star}$ English elements.

In contrast to the asymmetric-bilateral TIG in Case study 1, the two-hour leisure conversation examined in Case study 2 (Pitzl in press) contains elements from a total of ten *languages that are uttered by the six main interactants. While overt elements of the local *language (i.e. * German, since the interaction took place in Vienna) make up the majority of these elements (i.e. over two thirds), the remaining 30\% comprise elements from all other interactants' L1s (i.e. *Danish, ${ }^{\star}$ Dutch, ${ }^{\star}$ Norwegian, ${ }^{\star}$ Polish, ${ }^{\star}$ Spanish $/{ }^{\star}$ Catalan) as well as some additional isolated switches to other ${ }^{\star}$ languages (i.e. ${ }^{\star}$ Czech, ${ }^{\star}$ French, ${ }^{\star}$ Italian, ${ }^{\star}$ Latin). Notably, L1 elements that are introduced by one speaker are accommodated (here: converged) to and tend to be repeated verbatim by at least one other interlocutor, for whom they are, of course, non-L1 elements.

The micro-diachronic charts and the discussion of conversational transcripts in Pitzl (in press) show how diverse multilingual elements (i.e. elements from different *languages) tend to cluster in some stretches of the interaction throughout this long informal multi-party conversation. This clustering is most prominent in short episodes in which students teach each other to say 'cheers' in different *languages. Although this is a fairly mundane activity, it demonstrates how multilingual resources from individual speakers' IMRs are actively shared with interlocutors at different $\mathrm{T}_{1}$ points. Through instances of sharing (i.e. use in interaction), multilingual resources of individual participants 'migrate' to other interlocutors' IMRs (i.e. interlocutors learn these isolated non- ${ }^{\star}$ English elements in interaction). In this way, the non-`English words become shared multilingual resources in the 
central area of the TIG's MRP and are henceforth available to speakers in this particular TIG at various $\mathrm{T}_{2}$ points. By means of interaction, both IMRs and MRP expand over time (as schematically depicted in Figure 1 in Pitzl 2018b).

As can be seen by comparing the micro-diachronic development of the two TIGs in Case study 1 and 2, this process happens in similar, but nonetheless group-specific ways. This is because what can actually be shared at various $\mathrm{T}_{1}$ points throughout an interaction depends partly on the specific TIG constellation (such as the degree of diversity of speakers' IMRs and the resulting MRP) and partly on the micro-diachronic progress of the interaction. What has been shared (i.e. used and understood) in a TIG at a specific T1 point can subsequently (i.e. at various $\mathrm{T}_{2}$ points) be drawn upon in this TIG as an available multilingual resource, even if it was unknown to interlocutors when the interaction in this group started (i.e. at $\mathrm{T}_{0}$ ).

Adopting a process and progress perspective, micro-diachronic analysis also opens up the potential for describing situational norm development. Research on the emergence of multilingual practices and on communication in transient groups more generally raises fundamental questions about the nature of norms in language use, sociolinguistics and also pragmatics. Creativity needs norms as a point of reference in order to be identifiable (i.e. 'different from something'). Yet, norms (as well as judgements about creativity) are, in fact, analytic constructs that are neither stable nor transferable across time nor generalisable across space (i.e. they are not applicable to different contexts in the same way). If we take the implications of this seriously, empirical descriptions of any communicative phenomenon in transcultural contexts should be less focused on identifying (assumed) pre-existing linguistic norms and instead seek ways to trace, visualise and empirically describe how speakers jointly develop situationally appropriate practices - and how (new) situational norms might sometimes be posited as a result of these. For the use of multilingual resources (see e.g. Cogo 2018), this could mean to think of initial instances of code-switching and subsequent multilingual practices (if these manifest in an interaction) as first micro-diachronic 'stages' or phases of transient language contact. Crucially, as shown in Case study 2 and also Case study 3 below, such phases might be observable in fairly short synchronic data sets, such as one or two hours of naturally-occurring interaction.

An overarching phenomenon or process that plays a central role in this respect is accommodation (see e.g. Giles, Coupland, and Coupland 1991; Gasiorek, Giles, and Soliz 2015; Giles 2016). A group of international students may establish multilingual practices concerning multilingual cheers through repeated instances of convergence (such as verbatim other-repetition) as in Case study 2. In some contexts, this may lead to a point at which these practices solidify in the course of weeks and months so that local multilingual norms that can be reported 
by participants of a specific community of practice (see interviews e.g. in Kalocsai 2014). Yet, as illustrated by Case study 3, convergence towards emerging multilingual practices or group norms does not necessarily need to be inclusive.

\subsection{Case study 3: Convergence towards exclusive L1 side sequences in a bilateral business TIG}

The micro-diachronic analysis of what can be thought of as a symmetric-bilateral business TIG in Case study 3 (Pitzl 2021) examines how representatives of two business companies gradually reach an implicit, unspoken agreement that L1 use in side sequences is an acceptable - albeit 'exclusive' - multilingual practice in their TIG. Bringing together participants from Asia and Europe with typologically very different and mutually unintelligible L1s ( ${ }^{\star}$ German and ${ }^{\star}$ Korean), the meeting analysed in this study demonstrates how convergence may manifest quite subtly in the increasing use of unintelligible L1 side sequences as the interaction progresses throughout a three-and-a-half-hour business meeting. An earlier version of this analysis was carried out for a previous publication (Pitzl 2016b). Although this earlier analysis identified the same progression, it only relied on more established methodologies for data presentation (like the discussion of data excerpts) that is typically found in CA, interactional sociolinguistics and many ELF publications (cf. Section 3). The expanded analysis of Case study 3 in Pitzl (2021) complements such a conversational view (Pitzl 2021: 111) with novel quantitative and qualitative micro-diachronic research tools. Holistic overviews and micro-diachronic charts are used to trace and visualise the use of L1 ${ }^{\star}$ Korean and ${ }^{\star}$ German throughout the entire business meeting (Pitzl 2021). In this way, Case study 3 demonstrates how the expansion of traditional conversation and discourse analytic tools by means of a micro-diachronic approach enhances the detail of empirical description and can help us provide concrete evidence for the emergence of what might be called a situational multilingual etiquette.

Crucially, from a transcultural pragmatic perspective, Case study 3 illustrates that such a jointly developed, group-specific multilingual etiquette does not necessarily need to be inclusive. That is to say, although TIGs in many ELF contexts gravitate towards the use of shared and inclusive multilingual elements (as in Case study 1 and 2 and many ELF publications), unintelligible L1 use in short side-sequences (as in Case study 3) can also become a situational multilingual practice, yet an exclusive one. What is important from a transcultural pragmatic perspective is that a TIG does not simply 'have' or 'exhibit' multilingual practices, but that interactants always need to jointly develop and agree upon what they 
consider appropriate multilingual and pragmatic practices in their particular context.

In Case study 3, representatives of two companies meet for the first time $\left(\mathrm{T}_{0}\right)$. This makes building a good business relationship and establishing rapport key aims of their meeting. Thus, social aspects are important alongside the need to exchange transactional business content. Repeated unintelligible use of ${ }^{\star}$ German and Korean by the two parties in this meeting (i.e. at a very early stage of their business relationship) has the potential to cause mistrust. A micro-diachronic approach to the analysis of group interaction demonstrates that, at the beginning of their meeting, the participants do not take for granted that it is 'okay' to converse with colleagues in your L1 for longer periods of time. This practice only gradually develops throughout the meeting. Thus, implicit and/or explicit agreement on group-specific transcultural pragmatic conventions in TIGs always happens by means of and throughout interaction. If we are prepared to make the conceptual shift from intercultural to transcultural pragmatics, we are in need of methodologies that allow us to make this process fully visible in empirical description and data analysis. The micro-diachronic approach to spoken interaction employed in Case studies 1, 2 and 3 seeks to accomplish just that.

\section{Conclusion and outlook: Combining perspectives}

Taking the pragmatics of ELF communication as focal point of interest, this chapter has examined different ${ }^{\star}$ cultural approaches to the study of pragmatics and explored methodologies that are closely associated with these different approaches. Starting with cross-cultural approaches, Section 2 discussed how pragmatics with a cross-cultural orientation tends to draw on methods like DCTs to enable comparisons of different *languages or contrast so-called non-native/ learner/L2 pragmatic behaviour with the 'target' of native/L1 use. Section 3 then turned to intercultural pragmatics as the study of interactions among multilingual speakers, i.e. the perspective most prominent in research on ELF pragmatics. It linked an intercultural pragmatic perspective to salient methodologies that allow us to research interactions, in particular interactional sociolinguistics, conversation analysis (CA) and corpus linguistics. Section 4 then turned to current discourses on multilingualism and the distinction between inter- and transcultural communication in relation to the study of Transient International Groups (TIGs). Building on these discourses, it argued that the study of most ELF interactions should actually entail a shift from inter- to transcultural pragmatics and that this 
terminological and conceptual shift should have direct implications for methodology. It was suggested that a micro-diachronic approach to analysing (spoken) interaction would allow a more thorough empirical description of linguistic and pragmatic transcultural conventions as these emerge in real-time interaction.

To illustrate the potential of this micro-diachronic methodology, Section 5 summarised three empirical case studies, which describe the development of group-specific multilingual practices in different ELF-TIGs (drawing on VOICE data). These three case studies indicate that different TIG constellations, i.e. differences in group size or in bilateralness vs. multilingual diversity of a group, may have implications for the development of situational multilingual practices. That is to say, transcultural (E)LF encounters may involve the use of overt multilingual resources, but which specific multilingual patterns evolve in a TIG (e.g. inclusive vs. exclusive, predominance of one *language vs. use of many different *languages) will be situation- and group-specific.

Even more relevant to the study of transcultural pragmatics, these three descriptive case studies demonstrate that multilingual practices do not simply 'exist' in transient groups of multilingual speakers from the beginning (i.e. when speakers first meet), but are jointly developed by means of and throughout interaction. While this finding is not in itself 'new' and supported by evidence in many ELF publications, the level of qualitative and quantitative empirical detail that can be obtained by adopting a micro-diachronic approach to data analysis can be considered novel. A micro-diachronic approach to analysing (spoken) interaction opens up the possibility of describing transcultural pragmatics - and the situational development of transcultural communication more generally - in much more detail than has been done so far. Although this methodology is still in its early stages and is continuously being expanded, it is hoped that the present chapter has demonstrated its research potential for transcultural pragmatics, the study of TIGs, (English as a) lingua franca interactions and beyond.

A particular asset of micro-diachronic analysis is that it explicitly encourages the combination of different perspectives. With regard to methodology, this means a combination of distinct methodological traditions like CA, corpus linguistics, interactional sociolinguistics, discourse analysis and pragmatics. It also means the integration of qualitative data representation and detailed interpretation with select quantitative (or better: holistic) information, although notably statistical significance of quantifications is not a concern. Because of its adaptability to different research interests (i.e. pragmatic, multilingual, lexical, syntactic, etc.), micro-diachronic analysis also has the potential to allow the principled combination of different research foci on the same interaction. Quite clearly, processes like accommodation never only take place with regard to one phenomenon in a particular interaction, which makes it desirable to examine how the 
same group of speakers converge and/or diverge with regard to different aspects of language use and communication. As the research tools of micro-diachronic analysis continue to become more fleshed out, it will become possible to combine micro-diachronic portraits of one phenomenon (such as the emergence of multilingual practices, cf. Section 5) with the interactional development of different phenomena (such as negotiation of meaning, backchanneling or expressing disagreement) in the same data set (i.e. in the same TIG). In this way, an expansion of research methodology towards micro-diachronic analysis will allow for more in-depth portraits of transcultural communication, which will provide us with a more sophisticated understanding of the situational evolvement of transcultural pragmatic conventions.

\section{References}

Aijmer, Karin. 2020. Contrastive pragmatics and corpora. Contrastive Pragmatics 1(1). 28-57.

Austin, John L. 1975. How to do things with words, 2nd edn. Oxford: Clarendon.

Baker, Will. 2015. Culture and identity through English as a lingua franca: Rethinking concepts and goals in intercultural communication. Boston: De Gruyter Mouton.

Baker, Will. 2018. English as a lingua franca and intercultural communication. In Jennifer Jenkins, Will Baker \& Martin Dewey (eds.), The Routledge handbook of English as a lingua franca, 25-36. London: Routledge.

Baker, Will \& Chittima Sangiamchit. 2019. Transcultural communication: language, communication and culture through English as a lingua franca in a social network community. Language and Intercultural Communication 5(9). 1-17.

Bardovi-Harlig, Kathleen. 2010. Exploring the pragmatics of interlanguage pragmatics: Definition by design. In Anna Trosborg (ed.), Pragmatics across languages and cultures, 219-259. Berlin: De Gruyter Mouton.

Bjørge, Anne K. 2010. Conflict or cooperation: The use of backchannelling in ELF negotiations. English for Specific Purposes 29(3). 191-203.

Björkman, Beyza. 2017. PhD supervision meetings in an English as a lingua franca (ELF) setting: Linguistic competence and content knowledge as neutralizers of institutional and academic power. Journal of English as a Lingua Franca 6(1). 111-139.

Blackledge, Adrian \& Angela Creese. 2020. Heteroglossia. In Karin Tusting (ed.), The Routledge handbook of linguistic ethnography, 97-108. Abingdon: Routledge.

Blommaert, Jan. 2010. The sociolinguistics of globalization. Cambridge: Cambridge University Press.

Blommaert, Jan \& Ad Backus. 2011. Repertoires revisited: 'Knowing language' in superdiversity. Working Papers in Urban Language \& Literacies 67. 1-26.

Blum-Kulka, Shoshana \& Elite Olshtain. 1984. Requests and apologies: A cross-cultural study of speech act realization patterns (CCSARP). Applied Linguistics 5(3). 196-213.

Breiteneder, Angelika, Theresa Klimpfinger, Stefan Majewski \& Marie-Luise Pitzl. 2009. The Vienna-Oxford International Corpus of English (VOICE). A linguistic resource for exploring English as a lingua franca. ÖGAI Journal 28(1). 21-26. 
Busch, Brigitta. 2012. The linguistic repertoire revisited. Applied Linguistics 33(5). 503-523.

Busch, Brigitta. 2017. Expanding the notion of the linguistic repertoire: On the concept of Spracherleben -The lived experience of language. Applied Linguistics 38(3). 340-358.

Canagarajah, Suresh. 2013. Translingual practice: Global Englishes and cosmopolitan relations. Milton Park: Routledge.

Carey, Ray. 2013. On the other side: Formulaic organizing chunks in spoken and written academic ELF. Journal of English as a Lingua Franca 2(2). 207-228.

Cogo, Alessia. 2009. Accommodating difference in ELF conversations: A study of pragmatic strategies. In Anna Mauranen \& Elina Ranta (eds.), English as a lingua franca: Studies and findings, 254-273. Newcastle upon Tyne: Cambridge Scholars Publishing.

Cogo, Alessia. 2012. ELF and super-diversity: a case study of ELF multilingual practices from a business context. Journal of English as a Lingua Franca 1(2). 287-313.

Cogo, Alessia. 2016a. 'They all take the risk and make the effort': Intercultural accommodation and multilingualism in a BELF community of practice. In Lucilla Lopriore \& Enrico Grazzi (eds.), Intercultural communication. New perspectives from ELF, 365-383. Rome: Roma Tre Press.

Cogo, Alessia. 2016b. Visibility and absence: Ideologies of 'diversity' in BELF. In Marie-Luise Pitzl \& Ruth Osimk-Teasdale (eds.), English as a lingua franca: Perspectives and prospects. Contributions in honour of Barbara Seidlhofer (Trends in applied linguistics 24), 39-48. Boston: De Gruyter Mouton.

Cogo, Alessia. 2018. ELF and multilingualism. In Jennifer Jenkins, Will Baker \& Martin Dewey (eds.), The Routledge handbook of English as a lingua franca, 357-368. London: Routledge.

Cogo, Alessia. 2021. ELF and translanguaging. Covert and overt resources in a transnational workplace. In Kumiko Murata (ed.), ELF research methods and approaches to data and analyses: Theoretical and methodological underpinnings, 38-54. London: Routledge.

Cogo, Alessia \& Martin Dewey. 2012. Analyzing English as a lingua franca: A corpus-driven investigation. London: Continuum.

Cogo, Alessia \& Marie-Luise Pitzl. 2016. Pre-empting and signalling non-understanding in ELF. ELT Journal 70.1-7.

Cohen, Andrew D. 2018. Learning pragmatics from native and nonnative language teachers. Blue Ridge Summit: Multilingual Matters.

Creese, Angela \& Adrian Blackledge. 2010. Translanguaging in the bilingual classroom: A pedagogy for learning and teaching? The Modern Language Journal 94. 103-115.

Cutting, Joan. 2015. Pragmatics. A resource book for students, 3rd edn. Milton Park: Routledge.

Dorn, Nora, Martina Rienzner, Brigitta Busch \& Anita Santner-Wolfartsberger. 2014. "Here I find myself to be judged": ELF/plurilingual perspectives on language analysis for the determination of origin. Journal of English as a Lingua Franca 3(2). 409-424.

García, Ofelia \& Wei Li. 2014. Translanguaging: Language, bilingualism and education. Basingstoke: Palgrave Macmillan.

Gasiorek, Jessica, Howard Giles \& Jordan Soliz. 2015. Accommodating new vistas. Language \& Communication 41(1). 1-5.

Giles, Howard. 2016. The social origins of CAT. In Howard Giles (ed.), Communication accommodation theory, 1-12. Cambridge: Cambridge University Press.

Giles, Howard, Justine Coupland \& Nikolas Coupland (eds.). 1991. Contexts of accommodation: Developments in applied sociolinguistics (Studies in Emotion and Social Interaction). Cambridge: Cambridge University Press.

Goffman, Erving. 1981. Forms of talk. Philadelphia: University of Pennsylvania Press. 
Golato, Andrea. 2003. Studying compliment responses: A comparison of DCTs and recordings of naturally occurring talk. Applied Linguistics 24(1). 90-121.

Grice, Paul. 1989. Studies in the way of words. Cambridge: Harvard University Press.

Guido, Maria G. 2012. ELF authentication and accommodation strategies in crosscultural immigration encounters. Journal of English as a Lingua Franca 1(2). 219-240.

Guido, Maria G. 2016. ELF in responsible tourism: Power relationships in unequal migration encounters. In Marie-Luise Pitzl \& Ruth Osimk-Teasdale (eds.), English as a lingua franca: Perspectives and prospects. Contributions in honour of Barbara Seidlhofer, 49-56. Boston: De Gruyter Mouton.

Gumperz, John J. 1982. Discourse strategies. Cambridge: Cambridge University Press.

Gumperz, John J. 1999. On interactional sociolinguistic method. In Srikant Sarangi \& Celia Roberts (eds.), Talk, work and institutional order: Discourse in medical, mediation and management settings, 453-471. Berlin: Mouton de Gruyter.

Holliday, Adrian. 1999. Small cultures. Applied Linguistics 20(2). 237-264.

Holliday, Adrian, Martin Hyde \& John Kullman. 2004. Intercultural communication: An advanced resource book. London: Routledge.

Hülmbauer, Cornelia. 2016. Multi, pluri, trans ... and ELF: Lingualisms, languaging and the current lingua franca concept. In Marie-Luise Pitzl \& Ruth Osimk-Teasdale (eds.), English as a lingua franca: Perspectives and prospects. Contributions in honour of Barbara Seidlhofer, 193-203. Boston: De Gruyter Mouton.

Hülmbauer, Cornelia \& Barbara Seidlhofer. 2013. English as a lingua franca in European multilingualism. In Anne-Claude Berthoud, François Grin \& Georges Lüdi (eds.), Exploring the dynamics of multilingualism: The DYLAN project, 387-406. Amsterdam \& Philadelphia: Benjamins.

Hynninen, Niina, Kaisa S. Pietikäinen \& Svetlana Vetchinnikova. 2017. Multilingualism in English as a lingua franca: Flagging as an indicator of perceived acceptability and intelligibility. In Arja Nurmi, Tanja Rütten \& Päivi Pahta (eds.), Challenging the myth of monolingual corpora (Language and Computers: Studies in Digital Linguistics 80), 95-126. Leiden \& Boston: Brill/Rodopi.

Jenks, Christopher. 2013. 'Your pronunciation and your accent is very excellent': Orientations of identity during compliment sequences in English as a lingua franca encounters. Language and Intercultural Communication 13(2). 165-181.

Jenkins, Jennifer. 2015. Repositioning English and multilingualism in English as a lingua franca. Englishes in Practice 2(3). 49-85.

Kalocsai, Karolina. 2014. Communities of practice and English as a lingua franca: $A$ study of Erasmus students in a Central European context. Boston: De Gruyter Mouton.

Kappa, Katherine. 2019. Emergence in a transient social configuration: A linguistic ethnographic study of how strangers establish practices for working together within international development. Copenhagen: University of Copenhagen PhD dissertation.

Kaur, Jagdish. 2011. Raising explicitness through self-repair in English as a lingua franca. Journal of Pragmatics 43(11). 2704-2715.

Kaur, Jagdish. 2012. Saying it again: Enhancing clarity in English as a lingua franca (ELF) talk through self-repetition. Text \& Talk 32(5). 593-613.

Kaur, Jagdish. 2016. Conversation analysis and ELF. In Marie-Luise Pitzl \& Ruth Osimk-Teasdale (eds.), English as a lingua franca: Perspectives and prospects. Contributions in honour of Barbara Seidlhofer, 161-168. Boston: De Gruyter Mouton. 
Kaur, Jagdish. 2021. Applying conversation analysis to ELF interaction data. In Kumiko Murata (ed.), ELF research methods and approaches to data and analyses: Theoretical and methodological underpinnings, 161-178. London: Routledge.

Kirkpatrick, Andy. 2016. The Asian Corpus of English - introduction to the special issue. Journal of English as a Lingua Franca 5(2). 225-228.

Konakahara, Mayu. 2015. An analysis overlapping questions in casual ELF conversation: Cooperative or competitive contribution. Journal of Pragmatics 84. 37-53.

Kramsch, Claire. 2020. "I hope you can let this go"/ "Ich hoffe, Sie können das fallen lassen" - Focus on the perlocutionary in contrastive pragmatics. Contrastive Pragmatics 1(1). 58-81.

Li, Wei. 2018. Translanguaging as a Practical Theory of Language. Applied Linguistics 39(1). 9-30.

Love, Robbie, Claire Dembry, Andrew Hardie, Vaclav Brezina \& Tony McEnery. 2017. Compiling and analysing the Spoken British National Corpus 2014. International Journal of Corpus Linguistics 22(3). 319-344.

Matsumoto, Yumi. 2018. Functions of laughter in English-as-a-lingua-franca classroom interactions: A multimodal ensemble of verbal and nonverbal interactional resources at miscommunication moments. Journal of English as a Lingua Franca 7(2). 229-260.

Mauranen, Anna. 2003. The corpus of English as lingua franca in academic settings. TESOL Quarterly 37(3). 513-527.

Mauranen, Anna. 2006. A rich domain of ELF - The ELFA corpus of academic discourse. Nordic Journal of English Studies 5(2). 145-160.

Mauranen, Anna. 2012. Exploring ELF. Academic English shaped by non-native speakers. Cambridge: Cambridge University Press.

Mauranen, Anna. 2016. ELF corpora: Design, difficulties and triumphs. In Marie-Luise Pitzl \& Ruth Osimk-Teasdale (eds.), English as a lingua franca: Perspectives and prospects. Contributions in honour of Barbara Seidlhofer, 19-29. Boston: De Gruyter Mouton.

Metsä-Ketelä, Maria. 2006. 'Words are more or less superfluous': The case of more or less in academic lingua franca English. Nordic Journal of English Studies 5(2). 117-143.

Metsä-Ketelä, Maria. 2016. Pragmatic vagueness: Exploring general extenders in English as a lingua franca. Intercultural Pragmatics 13(3). 325-351.

Mortensen, Janus. 2017. Transient Multilingual Communities as a field of investigation: Challenges and opportunities. Journal of Linguistic Anthropology 27(3). 271-288.

Mortensen, Janus \& Spencer Hazel. 2017. Lending bureaucracy voice: Negotiating English in institutional encounters. In Markku Filppula, Juhani Klemola, Anna Mauranen \& Svetlana Vetchinnikova (eds.), Changing English: Global and local perspectives, 255-276. Berlin: De Gruyter Mouton.

Mujagic, Dajana. 2021. "Huge chaos and "syntactic nightmares": Exploring hyperbole in English as a lingua franca online group chats. Vienna: University of Vienna unpublished MA thesis.

Osimk-Teasdale, Ruth. 2014. "I just wanted to give a partly answer": Capturing and exploring word class variation in ELF data. Journal of English as a Lingua Franca 3(1). 109-143.

Pennycook, Alastair. 2007. Global Englishes and transcultural flows. London: Routledge.

Pietikäinen, Kaisa S. 2014. ELF couples and automatic code-switching. Journal of English as a Lingua Franca 3(1). 1-26.

Pietikäinen, Kaisa S. 2018. Silence that speaks: The local inferences of withholding a response in intercultural couples' conflicts. Journal of Pragmatics 129. 76-89. 
Pietikäinen, Kaisa S. 2021. Analysing multilingual/lingua franca interactions using conversation analysis: Notes on transcription and representability. In Kumiko Murata (ed.), ELF research methods and approaches to data and analyses: Theoretical and methodological underpinnings, 179-196. London: Routledge.

Pitzl, Marie-Luise. 2005. Non-understanding in English as a lingua franca: Examples from a business context. Vienna English Working Papers 14(2). 50-71.

Pitzl, Marie-Luise. 2010. English as a lingua franca in international business: Resolving miscommunication and reaching shared understanding. Saarbrücken: VDM.

Pitzl, Marie-Luise. 2015. Understanding and misunderstanding in the Common European Framework of Reference: What we can learn from research on BELF and intercultural communication. Journal of English as a Lingua Franca 4(1). 91-124.

Pitzl, Marie-Luise. 2016a. World Englishes and creative idioms in English as a lingua franca. World Englishes 35(2). 293-309.

Pitzl, Marie-Luise. 2016b. Investigating multilingual practices in BELF meetings with VOICE: A corpus linguistic case study with methodological considerations. Waseda Working Papers in ELF 5. 15-40.

Pitzl, Marie-Luise. 2017. Communicative 'success', creativity and the need for de-mystifying L1 use: Some thoughts on ELF and ELT. Lingue e Linguaggi 24. 37-46.

Pitzl, Marie-Luise. 2018a. Creativity in English as a lingua franca: Idiom and metaphor. Boston: Mouton de Gruyter.

Pitzl, Marie-Luise. 2018b. Transient International Groups (TIGs): Exploring the group and development dimension of ELF. Journal of English as a Lingua Franca 7(1). 25-58.

Pitzl, Marie-Luise. 2019. Investigating Communities of Practices (CoPs) and Transient International Groups (TIGs) in BELF contexts. Iperstoria 13. 5-14.

Pitzl, Marie-Luise. 2021. Tracing the emergence of situational multilingual practices in a BELF meeting: Micro-diachronic analysis and implications of corpus design. In Kumiko Murata (ed.), ELFresearch methods and approaches to data and analyses: Theoretical and methodological underpinnings, 97-125. London: Routledge.

Pitzl, Marie-Luise. in press. Multilingual creativity and emerging norms in interaction: Towards a methodology for micro-diachronic analysis. In Janus Mortensen \& Kamilla Kraft (eds.), Norms and the study of language in social life. Berlin: De Gruyter.

Radinger, Sandra. 2018. Language awareness and agency in the availability of linguistic resources. A case study of refugees and locals in Austria. Language Awareness 27(1-2). 61-78.

Ranta, Elina. 2006. The 'attractive' progressive - Why use the -ing form in English as a lingua franca? Nordic Journal of English Studies 5(2). 97-116.

Ranta, Elina. 2018. Grammar in ELF. In Jennifer Jenkins, Will Baker \& Martin Dewey (eds.), The Routledge handbook of English as a lingua franca, 244-254. London: Routledge.

Santner-Wolfartsberger, Anita. 2015. Parties, persons, and one-at-a-time: Conversation analysis and ELF. Journal of English as a Lingua Franca 4(2). 253-282.

Sarangi, Srikant. 1994. Intercultural or not? Beyond celebration of cultural differences in miscommunication analysis. Pragmatics 4(3). 409-427.

Schmid, Hans-Jörg. 2020. The dynamics of the linguistic system: Usage, conventionalization, and entrenchment. Oxford: Oxford University Press.

Scollon, Ron \& Suzanne W. Scollon. 1995. Intercultural communication: A discourse approach. Oxford: Blackwell.

Searle, John R. 1979. Expression and meaning: Studies in the theory of speech acts. Cambridge: Cambridge University Press. 
Seidlhofer, Barbara. 2001. Closing a conceptual gap: The case for a description of English as a lingua franca. International Journal of Applied Linguistics 11(2). 133-158.

Seidlhofer, Barbara. 2010. Giving VOICE to English as a lingua franca. In Roberta Facchinetti, David Crystal \& Barbara Seidlhofer (eds.), From international to local English - and back again, 147-163. Bern \& New York: Peter Lang.

Seidlhofer, Barbara. 2011. Understanding English as a lingua franca. Oxford: Oxford University Press.

Seidlhofer, Barbara. 2012. Corpora and English as a lingua franca. In Ken Hyland, Chau M. Huat \& Michael Handford (eds.), Corpus applications in applied linguistics, 135-149. London: Bloomsbury.

Seidlhofer, Barbara. 2013. Corpus analysis of English as a lingua franca. In Carol A. Chapelle (ed.), The encyclopedia of applied linguistics, 1-5. London: Blackwell.

Seidlhofer, Barbara. 2021. Researching ELF communication. Focus on high-stakes encounters. In Kumiko Murata (ed.), ELF research methods and approaches to data and analyses: Theoretical and methodological underpinnings, 29-37. London: Routledge.

Spencer-Oatey, Helen. 2002. Managing rapport in talk: Using rapport sensitive incidents to explore the motivational concerns underlying the management of relations. Journal of Pragmatics 34. 529-545.

Thomas, Jenny. 1983. Cross-cultural pragmatic failure. Applied Linguistics 4(2). 91-112.

Vertovec, Steven. 2007. Super-diversity and its implications. Ethnic and Racial Studies 30(6). 1024-1054.

Vettorel, Paola. 2019. Communication strategies and co-construction of meaning in ELF: Drawing on "Multilingual Resource Pools". Journal of English as a Lingua Franca 8(2). 179-210.

VOICE. 2013. The Vienna-Oxford International Corpus of English (version 2.0 XML). Director: Barbara Seidlhofer; Researchers: Angelika Breiteneder, Theresa Klimpfinger, Stefan Majewski, Ruth Osimk-Teasdale, Marie-Luise Pitzl, Michael Radeka.

Wang, Lixun. 2021. Asian Corpus of English (ACE): Features and applications. In Kumiko Murata (ed.), ELF research methods and approaches to data and analyses: Theoretical and methodological underpinnings, 126-142. London: Routledge.

Widdowson, Henry. 2015. ELF and the pragmatics of language variation. Journal of English as a Lingua Franca 4(2). 359-372.

Widdowson, Henry. 2021. Research perspectives on ELF. Linguistic usage and communicative use. In Kumiko Murata (ed.), ELF research methods and approaches to data and analyses: Theoretical and methodological underpinnings, 21-28. London: Routledge.

Zhu Hua. 2011. Introduction. Themes and issues in the study of language and intercultural communication. In Zhu Hua (ed.), The language and intercultural communication reader, 1-14. Abingdon: Routledge.

Zhu Hua. 2014. Exploring intercultural communication: Language in action. London: Routledge.

Zhu Hua. 2015. Negotiation as the way of engagement in intercultural and lingua franca communication: Frames of reference and interculturality. Journal of English as a Lingua Franca 4(1). 63-90. 